Volume 19, No 3 International Journal of Radiation Research, July 2021

\title{
Observation of the clinical outcomes in Temozolomide and radiotherapy Combination against the metastatic brain tumors
}

\author{
J. Zhang, D. Zhang, Y. Li, C. Zhang, S. Yuan, B. Zhu* \\ Department of Neurosurgery, Sunshine Union Hospital of Shandong Province, Weifang, Shandong261000, China
}

\section{- Original article}

*Corresponding authors:
Bing Zhu, Ph.D.,
E-mail:
$\quad$ Drcorespond@gmail.com
Revised: July 2020
Accepted: July 2020
Int. J. Radiat. Res., July 2021;
19(3): 737-741
DOI: 10.29252/ijrr.19.2.737

\begin{abstract}
Background: To observe the efficacy of temozolomide in combination with radiotherapy against metastatic brain tumors. Materials and Methods: This study was a prospective, observational, open-label study, conducted in clinical practice implanted for metastatic brain tumor patients. A total of 106 patients with metastatic brain tumors were enrolled in this study, and according to the sequence of admission, they were randomized into the experiment group (temozolomide + radiotherapy) and the control group (radiotherapy). During the 24 months of follow-up, we compared the clinical efficacy, recurrence time, survival time and quality, and the adverse reactions of the patients between two groups. Results: Short-term remission after treatment was higher in the experimental group compared to control $(P<0.05)$. During the 24-month follow-up, we found that patients in the experiment group had longer recurrence time and survival time than their counterparts in the control group $(P<0.05)$. After treatment, the scores of the life quality of patients in the experiment group were better than those in the control group $(P<0.05)$. Also, there was a lower rate of the incidence of the adverse reactions in the experiment group $(P<0.05)$. Conclusion: For metastatic brain tumors, temozolomide in combination with radiotherapy works better as a safe and reliable strategy in prolonging the survival time, increasing life quality while reducing the adverse reactions. The strength of this study was evaluating the quality of life as an important outcome of the chemotherapy+radiotherapy regimen.
\end{abstract}

Keywords: Metastatic brain tumors, temozolomide, radiotherapy, clinical efficacy.

\section{INTRODUCTION}

Metastatic brain tumors, a group of common intracerebral tumors, are often featured by the high prevalence, mainly in the population aged between 20 and 50 years old (1). Metastatic brain tumors originate from a variety of primary tumors, including gastrointestinal tumors, nonsmall cell lung cancer, and breast cancer. As reported by the latest survey, the death of about $25 \%$ of cancers (2). Previously, surgical treatment was considered as the first option in clinical treatment. With the development of medical techniques, radiotherapy has become an ideal option in the treatment of metastatic brain tumors, with a high remission rate $(3,4)$.

Temozolomide (TMZ) is a kind of regular chemotherapeutics for the treatment of the intracerebral tumors in recent years in small molecular weight with a wide anti-tumor spectrum, and the ability to penetrate the blood-brain barrier. Thus, after oral administration of TMZ, the blood concentration of TMZ attains to $30 \%$ to $40 \%$ rapidly, with the bioavailability of about $100 \%$.

Moreover, TMZ is concentrated mainly in the site of tumors, showing potent efficacy and high selectivity, but little adverse effect $(5,6)$. While there are controversies among the studies assessing radiotherapy along with $\mathrm{TMZ}$, its 
efficiency is not fully investigated, and there is little data on its possible adverse effects; we investigated the efficacy of the combination of TMZ and radiotherapy for treatment of metastatic brain tumors, and detailed information is reported as follows.

\section{MATERIALS AND METHODS}

\section{General material}

This study was a prospective, observational, open-label study, conducted in clinical practice implanted for metastatic brain tumor patients. A total of 106 patients with metastatic brain tumors were enrolled in this study based on the simply available sampling. The inclusion criteria were the confirmed diagnosis of primary tumors by CT or MRI. Patients had primary tumors of gastrointestinal tumors, non-small cell lung cancer, and breast cancer. Exclusion criteria were the patient's disagreement to continue the study and not referring to the follow-ups. Before the implementation of this study, patients or their families signed the informed consent, and the study was approved by the ethics committee of the Sunshine Union Hospital of Shandong Province based on the Helsinki statement. According to the sequence of admission, they were randomized into the experiment group (temozolomide + radiotherapy) and the control group (radiotherapy) by random numbers method. In the experiment group, there were 36 males and 17 females, aged between 30 and 74 years old, with an average of (52.8 \pm 5.6$)$ years old; 23 patients were diagnosed as breast cancer, 17 as colorectal cancer and 13 as lung cancer.

In the control group, there were 34 males and 19 females, aged from 30 to 74 years old, with an average of (52.9 \pm 5.5$)$ years old; 24 patients were diagnosed as breast cancer, 16 as gastrointestinal cancer, and 13 as non-small cell lung cancer. No statistical significance in differences between the general data was shown in the comparison of the general data $(P=0.05)$.

\section{Methods}

Patients in the control group only took the 738 radiotherapy: Anterior posterior-posterior anterior whole-brain radiation was performed (Elekta radiotherapy device; Sweden), with a radiation dose of 4000 cGy being delivered in 20 fractions at 200 cGy per fraction, five days per week over 4 weeks. For those in the experiment group, they would additionally take the TMZ capsules (Jiangsu Tasly Diyi Pharmaceutical Co., Ltd; Lot No.: 130621) on the second day after radiotherapy via oral administration at $75 \mathrm{mg} /$ $\left(\mathrm{m}^{2} \cdot \mathrm{d}\right)$, twice per day over 4 weeks.

During radiation, changes in the condition of patients were monitored closely, including the symptoms of nausea, vomiting, and an increase in blood pressure, or edema. Furthermore, additional attention should be paid towards the increase in the intracerebral pressure and the progression in condition. Mannitol and glycerin fructose were advised for patients, and the symptomatic measures were immediately taken for the adverse reactions and efficacy. Antiemetics were taken according to the medical advice before the TMZ administration to mitigate the gastrointestinal reaction.

Patients were advised to take the high-protein, high-vitamin but digestible food, and, if necessary, the biologicals to improve the immunity. During radiation or administration of TMZ, patients might suffer from hair loss, skin itch, or the increased scurf, and accordingly, they were advised to wear the hat when they went outside. Stimulus shampoo was strongly prohibited, and so did the scrubbing the itchy site. Different strategies were taken to overcome the fear of the patients or their family, to help them establish confidence and cooperate with the treatment.

\section{Observation index and criteria for efficacy evaluation \\ Short-term efficacy}

Complete remission (CR): Tumor disappearance for over 4 weeks, and no emergence of the new lesion after treatment;

Partial remission (PR): Tumor shrinkage by over $50 \%$ for 4 weeks or longer, and no emergence of new lesions after treatment;

Stable disease (SD): Tumor shrinkage by less than $50 \%$ for 4 weeks or longer, and no

Int. J. Radiat. Res., Vol. 19 No. 3, July 2021 
emergence of new lesions after treatment;

Progression in disease (PD): Tumor enlargement by $25 \%$ or more, or the emergence of new lesions.

Total remission rate $=(\mathrm{CR}+\mathrm{PR}) /$ total cases $\times 100 \%$.

\section{Long-term efficacy}

A 24-month follow-up was performed after treatment to observe the changes in the recurrence time and survival time of patients in two groups. Survival quality of patients before and after treatment was evaluated by using the Karnofaky methods, and a higher score represented the better survival quality. Karnofsky is a performance quality level assessment tool rating from zero to one hundred. Observing the incidence of adverse reactions of patients in two groups.

\section{Statistical analysis}

SPSS 19.0 software was adopted for the statistical analysis of this study. Chi-square test was carried out for the enumeration data, while $t$-test for the comparison of the measurement data in the form of mean \pm standard deviation. $P<4.49$ suggested the statistical significance of the difference.

\section{RESULTS}

\section{Comparison of the remission after treatment between two groups}

Statistical significance was shown in the difference when comparing the remission rates between two groups $(\mathrm{P}<0.05$; table 1$)$.

Table 1. Comparison of the short-term remission after treatment between two groups [n (\%)].

\begin{tabular}{|c|c|c|c|c|}
\hline Item & $\begin{array}{c}\text { Experiment } \\
\text { group }(\mathbf{n = 5 3 )})\end{array}$ & $\begin{array}{c}\text { Control } \\
\text { group }(\mathbf{n = 5 3 )}\end{array}$ & $\mathbf{c}^{\mathbf{2}}$ & $\boldsymbol{P}$ \\
\hline CR & $12(22.64)$ & $8(15.09)$ & 0.73 & 0.402 \\
\hline PR & $37(69.81)$ & $32(60.38)$ & 0.75 & 0.373 \\
\hline SD & $3(5.66)$ & $8(15.09)$ & 1.92 & 0.174 \\
\hline PD & $1(1.89)$ & $5(9.44)$ & 3.93 & 0.046 \\
\hline $\begin{array}{c}\text { Total } \\
\text { remission rate }\end{array}$ & $49(92.45)$ & $40(75.47)$ & 3.97 & 0.043 \\
\hline
\end{tabular}

Comparison of the long-term efficacy between two groups

During the 24-month follow-up after treatment, we found that patients in the experiment group had longer recurrence time and survival time than those in the control group, and the differences had statistical significance $(P / 0.05$; table 2$)$.

Table 2. Comparison of the long-term efficacy between two groups (Means \pm standard deviation, months).

\begin{tabular}{|c|c|c|c|}
\hline Group & $\mathbf{n}$ & Recurrence time & Survival time \\
\hline Experiment group & 53 & $3.1 \pm 0.5$ & $14.9 \pm 0.7$ \\
\hline Control group & 53 & $9.1 \pm 0.3$ & $10.6 \pm 0.5$ \\
\hline $\boldsymbol{t}$ & & 7.712 & 34.82 \\
\hline $\boldsymbol{P}$ & & 0.000 & 0.000 \\
\hline
\end{tabular}

Comparison of the survival quality scores between two groups

Before the treatment, no statistical significance was identified in the comparison of the life quality scores between two groups $(P=$ 0.05); but after treatment, life quality was somehow ameliorated in comparison with the condition of patients, and the amelioration in the experiment group was much better than that in the control group $(P / 0.05$; table 3$)$.

Table 2. Comparison of the long-term efficacy between two groups (Means \pm standard deviation, months).

\begin{tabular}{|c|c|c|c|c|c|}
\hline Group & $\mathbf{n}$ & $\begin{array}{c}\text { Before } \\
\text { treatment }\end{array}$ & $\begin{array}{c}\text { After } \\
\text { treatment }\end{array}$ & $\boldsymbol{t}$ & $\boldsymbol{P}$ \\
\hline $\begin{array}{c}\text { Experiment } \\
\text { group }\end{array}$ & 53 & $68.4 \pm 4.3$ & $83.4 \pm 2.3$ & 21.457 & 0.000 \\
\hline Control group & 53 & $69.3 \pm 4.6$ & $74.2 \pm 3.1$ & 6.145 & 0.000 \\
\hline$t$ & & & 0.925 & 15.64 & \\
\hline$P$ & & & 0.358 & 0.000 & \\
\hline
\end{tabular}

\section{Comparison of the adverse reactions between two groups}

Statistical significance was found in the difference in the incidence rate of adverse reactions between two groups $(\mathrm{P}<0.05$; table 4). 
Table 4. Comparison of the incidence of adverse reactions between two groups [n (\%)].

\begin{tabular}{|c|c|c|c|c|c|}
\hline Group & $\mathbf{n}$ & Headache & Vomiting and nausea & Bone marrow suppression & Total incidence rate \\
\hline Experiment group & 53 & $2(3.77)$ & $1(1.89)$ & $1(1.89)$ & $4(7.55)$ \\
\hline Control group & 53 & $6(11.32)$ & $4(7.55)$ & $3(5.66)$ & $13(24.53)$ \\
\hline $\mathbf{c}^{\mathbf{2}}$ & & & & & 4.93 \\
\hline $\boldsymbol{P}$ & & & & & 0.024 \\
\hline
\end{tabular}

\section{DISCUSSION}

Metastatic brain tumors are a common condition frequently seen in clinical practice and usually treated by conventional anti-tumor drugs or surgical treatment previously. However, due to the difficulty of chemotherapeutics in passing through the blood -brain-barriers into the central nervous system, they are less prominent in efficacy, but with the higher possibility to induce the adverse reactions (7). Moreover, the surgical treatment also has a variety of limitations, and the stringent requirement for the surgical indications are restricting the application scope in clinical practice ${ }^{(8)}$.

Currently, with the rapid development in medical techniques, radiotherapy has become one of the most frequent methods for the treatment of metastatic brain tumors. As reported, radiotherapy for the treatment of metastatic brain tumors prolongs the survival time of patients from 1 month to 3 to 6 months (9). However, for whole-brain radiotherapy, the limitation in the dose of radiotherapy curbs the efficacy of tumors (10). Thus, single radiotherapy is not an ideal choice for the treatment of metastatic brain tumors.

TMZ, a novel anti-tumor drug, is a kind of fat-soluble small-molecular alkylating agent with high specificity and the ability to pass through the blood-brain barrier. It was firstly applied in treatment of the malignant tumors, including malignant brain glioma and astrocytoma (11). Mechanistically, TMZ interferes with the DNA replication of tumor cells by methylation, thereby inhibiting the proliferation of tumor cells (12). Also, through oral administration, TMZ can be delivered directly to the central nervous system to increase blood concentration. Besides, TMZ is found to mitigate the clinical symptoms efficiently with fewer adverse reactions, which is conducive to the improvement of surgical tolerance in the treatment of metastatic brain tumors (13). Nevertheless, a study by Gamboa-Vignolle et al. (14) found no statistical significance in differences of the progression-free survival (PFS) or the total survival rate between the combined treatment group and the single radiotherapy, while the incidence rate of the adverse reactions in the combined treatment group was even higher than that in the single radiotherapy group (HR=2.03, 95\% CI: 1.5 - 2.64). Christodoulou et al. (15) indicated that the combined treatment of WBRT and TMZ performed well in the treatment of the metastatic brain tumors when comparing to the single treatment of TMZ (RR=1.34, $P<4.445)$, and the subgroup analysis also revealed that the objective response rate (ORR) of the NSCLC patients, instead of the breast cancer patients $(\mathrm{RR}=1.03, P \times 0.86)$, was significantly higher than that in the control group $(\mathrm{RR}=1.38$, $P<0.001$ ).

The results of this study showed that the difference in the remission rates of patients in two groups had statistical significance, coinciding with the conclusion reported by literature (16). During the 24-month follow-up, the recurrence time and survival time of patients in the experiment group were all longer than those in the control group, which is also consistent with the results of previous literature (17). Combined treatment of the TMZ and radiotherapy can prolong the recurrence time and survival time of patients, and gain the ideal long-term efficacy. Moreover, consistent with the results of the literature (18), amelioration of the survival quality of patients in the experiment group is much better, with fewer adverse reactions. Our study was limited due to being conducted in one center that had not a high number of metastatic brain tumor cases. So we suggest further researches with a higher sample

Int. J. Radiat. Res., Vol. 19 No. 3, July 2021 
size.

In conclusion, the strength of this study was evaluating the quality of life as an important outcome of the chemotherapy + radiotherapy regimen. Previous researches have just focused on the clinical outcomes and medical efficiency of this protocol. While our results of remission, Partial remission, Progression in disease, and Stable disease rate were consistent with the study of Hegi et al. (18) and Yu et al. (17); our results revealed its benefits to the total aspects of life pointed by the quality of life.

\section{Conflicts of interest: Declared none.}

\section{REFERENCES}

1. Mikuni $N$ and Miyamoto $S$ (2010) Surgical treatment for glioma: extent of resection applying functional neurosurgery. Neurol Med Chir (Tokyo), 50(9): 720-726.

2. Tang J, Zhou H, Hou X, Wang L, Li Y, Pang Y, Chen C, Jiang G, Liu Y (2018) Enhanced anti-tumor efficacy of temozolomide-loaded carboxyl -ated poly(amido-amine) combined with photothermal/photodynamic therapy for melanoma treatment. Cancer Letters, 423(3): 1-9.

3. Barbagallo GM, Paratore $S$, Caltabiano R, Palmucci S, Parra HS, Privitera G, Motta F, Lanzafame S, Scaglione G, Longo A, Albanese $V$ (2014) Long-term therapy with temozolomide is a feasible option for newly diagnosed glioblastoma: a single - institution experience with as many as 101 temozolomide cycles. Neurosurg Focus, 37(6): E4.

4. Candolfi M, Yagiz K, Wibowo M, Ahlzadeh GE, Puntel M, Ghiasi H, Kamran N, Paran C, Lowenstein PR, Castro MG (2014) Temozolomide does not impairgene therapy-mediated antitumor immunity in syngeneic brain tumormodels. Clin Cancer Res, 20(6): 1555-1565.

5. Chibbaro S, Benvenuti L, Caprio A, Carnesecchi S, Pulerà F, Faggionato F, Serino D, Galli C, Andreuccetti M, Buxton N, Gagliardi R (2004) Temozolomide as first-lineagent in treating high - grade gliomas: phase II study. I Neurooncol, 67(1-2): 77-81.

6. Kawaji H, Tokuyama T, Yamasaki T, Amano S, Sakai N, Namba H (2015) Interferon-beta and temozolomide combination therapy for temozolomide monotherapy-refractorymalignant gliomas. Mol Clin Oncol, 3(4): 909-913.

7. Koekkoek JA, Dirven L, Heimans JJ, Postma TJ, Vos MJ, Reijneveld JC, Taphoorn MJ (2016) Seizure reduction is a prognostic marker in low - grade glioma patients treated with temozolomide. J Neurooncol, 126(2): 347-354.

8. Syro LV, Uribe H, Penagos LC, Ortiz LD, Fadul CE, Horvath
E, Kovacs K (2006) Antitumour effects of temozolomide in a man with a large, invasive prolactin-producing pituitary neoplasm. Clinical Endocrinology, 65(4): 552-3.

9. Whitelaw BC, Dworakowska D, Thomas NW, Barazi S, Riordan-Eva P, King AP, Hampton T, Landau DB, Lipscomb D, Buchanan CR, Gilbert JA (2012) Temozolomide in the management of dopamine agonist-resistant prolactinomas. Clinical Endocrinology, 76(6): 877-86.

10. Deng $X$, Zheng Z, Lin B, Su H, Chen H, Fei S, Fei Z, Zhao L, Jin $X$, Xie CY (2017) The efficacy and roles of combining temozolomide with whole brain radiotherapy in protection neurocognitive function and improvement quality of life of non-small-cell lung cancer patients with brain metastases. BMC Cancer, 17(1): 42.

11. Annamalai AK, Dean AF, Kandasamy N (2012) Temozolomide responsiveness in aggressive corticotroph tumours: a case report and review of the literature. Pituitary, 15(3): 276-287.

12. Murakami M, Mizutani A, Asano S, Katakami H, Ozawa $Y$, Yamazaki K, Ishida Y, Takano K, Okinaga H, Matsuno A (2011) A mechanism of acquiring temozolomide resistance during transformation of atypical prolactinoma into prolactin-producing pituitary carcinoma: case report. Neurosurgery, 68(6e): 1761-1767.

13. Minniti G, Scaringi C, Lanzetta G, Bozzao A, Romano A, De Sanctis V, Valeriani M, Osti M, Enrici RM (2014) Whole brain reirradiation and concurrent temozolomide in patients with brain metastases. I Neurooncol, 118(2): 329334.

14. Gamboa-Vignolle C, Ferrari-Carballo T, Arrieta Ó, Mohar A (2012) Whole-brain irradiation with concomitant daily fixed - dose temozolomide forbrain? metastases treatment: a randomised phase II trial. Radiother Oncol, 102(2): $187-191$.

15. Christodoulou C, Bafaloukos D, Linardou H, Aravantinos G, Bamias A, Carina M, Klouvas G, Skarlos D (2005) Temozolomide (TMZ) combined with cisplatin (CDDP) in patients with brain metastases from solid tumors: a Hellenic Cooperative Oncology Group(HeCOG) Phase II study. J Neurooncol, 71(1): $61-65$.

16. He H, Yao M, Zhang W, Tao B, Liu F, Li S, Dong Y, Zhang C, Meng Y, Li Y, Hu G (2015) MEK2 is a prognostic marker and potential chemo- sensitizing target for glioma patients undergoing temozolomide treatment. Cell Mol Immunol, 20(7): 1036-1046.

17. Yu Z, Xie G, Zhou G, Cheng Y, Zhang G, Yao G, Chen Y, Li Y, Zhao G (2015) NVP - BEZ235, a novel dual PI3K - mTOR inhibitor displays anti - glioma activity and reduces chemoresistance to temozolomide in human glioma cells.

Cancer Lett, 367(1): 58-68.

18. Hegi ME, Diserens AC, Gorlia T, Hamou MF, De Tribolet N, Weller M, Kros JM, Hainfellner JA, Mason W, Mariani L, Bromberg JE (2005) MGMT gene silencing and benefit from temozolomide in glioblastoma. N Engl J Med, 352(10): 997-1003. 
